\title{
Neurohormonas y citocinas en la insuficiencia cardíaca. Correlación con la reserva de flujo coronario
}

\author{
Isabel Coma-Canella ${ }^{a}$, Alfonso Macías ${ }^{a}$, Nerea Varob y Alfonso Sánchez Ibarrolac \\ aServicio de Cardiología. Clínica Universitaria. Facultad de Medicina. Universidad de Navarra. Pamplona. España. \\ bServicio de Bioquímica. Clínica Universitaria. Facultad de Medicina. Universidad de Navarra. Pamplona. España. \\ 'Servicio de Inmunología. Clínica Universitaria. Facultad de Medicina. Universidad de Navarra. Pamplona. España.
}

Introducción y objetivos. La reserva del flujo coronario (RFC) medida con tomografía por emisión de positrones (PET) está disminuida en la insuficiencia cardíaca. Puesto que las neurohormonas y las citocinas también presentan modificaciones en estos pacientes, pretendemos comprobar si hay correlación entre la RFC y los valores de neurohormonas y citocinas.

Pacientes y método. Se estudió a 40 pacientes diagnosticados de cardiopatía no isquémica e insuficiencia cardíaca. El flujo miocárdico (FM) se midió mediante PET y N-13 amonio: en condiciones basales y durante la infusión de trifosfato de adenosina (ATP). En cada uno se calculó la RFC. En todos se determinaron la noradrenalina, la endotelina-1, angiotensina II, el péptido natriurético auricular (ANP) y cerebral (BNP), el factor de necrosis tumoral alfa, la interleucina (IL)-1 $\beta$ y el receptor soluble de IL-2 e IL-6.

Resultados. Todas las neurohormonas medidas tuvieron valores superiores a los de referencia. Las citocinas medidas también estuvieron elevadas, excepto la IL-1 3 . Se encontró una correlación negativa significativa entre la RFC y los siguientes factores: ANP $(r=-0,476)$, BNP $(r=$ $-0,442)$ e IL-6 ( $r=-0,509)$.

Conclusiones. En la insuficiencia cardíaca, la disminución de la RFC se correlaciona con el aumento de determinadas neurohormonas (ANP, BNP) y citocinas (IL-6), de efecto vasodilatador. Se trata, probablemente, de mecanismos de compensación insuficientes ante la disfunción endotelial que presentan estos enfermos.

Palabras clave: Insuficiencia cardíaca. Reserva de flujo coronario. Neurohormonas. Citocinas.

\section{Neurohormones and Cytokines in Heart Failure. Correlation With Coronary Flow Reserve}

Introduction and objectives. In heart failure, the coronary flow reserve (CFR) measured by positron-emission tomography (PET) is reduced. As neurohormone and cytokine levels are also altered in patients with the condition, our aim was to determine whether there is a correlation between CFR and neurohormone and cytokine levels.

Patients and method. The study included 40 patients with heart failure but without ischemic heart disease. Myocardial blood flow was measured by PET using nitrogen13 ammonia at baseline and during ATP infusion. The CFR was calculated for each patient. In addition, levels of the following were determined: norepinephrine, endothelin-1, angiotensin-II, atrial natriuretic peptide (ANP), brain natriuretic peptide (BNP), tumor necrosis factor-alpha, interleukin (IL)-1 $\beta$, soluble IL-2 receptor, and IL-6.

Results. All neurohormone levels were elevated above reference values. The levels of all cytokines, except IL-1 $\beta$, were also elevated. There was a significant negative correlation between CFR and the levels of several neurohormones: ANP $(r=-0.476)$, BNP $(r=-0.442)$, and IL-6 $(r=-0.509)$.

Conclusions. In heart failure, the decrease in CFR is correlated with increases in the levels of certain neurohormones (i.e., ANP and BNP) and cytokines (i.e., IL-6), with vasodilatory effect. These increases are probably are related to compensatory mechanisms that are unable to correct for the endothelial dysfunction present in these patients.

Key words: Heart failure. Coronary flow reserve. Neurohormones. Cytokines.

Full English text available at: www.revespcardiol.org

\section{INTRODUCCIÓN}

En un estudio previo ${ }^{1}$, nuestro grupo demostró por primera vez que la reserva de flujo coronario (RFC), medida mediante tomografía por emisión de positrones (PET), está reducida en la insuficiencia cardíaca
Correspondencia: Dra. I. Coma-Canella.

Departamento de Cardiología. Clínica Universitaria.

Avda. de Pío XII, 36. 31008 Pamplona. España.

Correo electrónico: icoma@unav.es

Recibido el 7 de febrero de 2005.

Aceptado para su publicación el 22 de junio de 2005. 


\section{ABREVIATURAS}

RFC: reserva de flujo coronario.

PET: tomografía por emisión de positrones.

ANP: péptido natriurético auricular.

BNP: péptido natriurético cerebral.

IL: interleucina.

no isquémica, cualquiera que sea su etiología. Además, los pacientes en peor clase funcional son los que tienen la reserva más alterada.

Por otra parte, numerosos estudios muestran que en la insuficiencia cardíaca se elevan distintas neurohormonas y citocinas $^{2-11}$. Entre ellas se pueden señalar las siguientes: noradrenalina, angiotensina II, endotelina1, péptido natriurético auricular (ANP) y cerebral (BNP), interleucina (IL)-1 $\beta$, IL-6, receptor soluble de la IL-2 y factor de necrosis tumoral alfa. Generalmente, la magnitud de su elevación tiene relación con el pronóstico.

El estudio actual tiene como objetivo establecer una posible correlación entre la RFC medida por PET y las neurohormonas y citocinas que han sido determinadas en estos mismos pacientes.

\section{PACIENTES Y MÉTODO}

\section{Pacientes}

Se incluyó en el estudio a 40 pacientes consecutivos (edad media, $63 \pm 11$ años; 27 varones), 30 procedentes de la consulta externa y 10 hospitalizados en el servicio de cardiología. Los criterios de inclusión fueron los siguientes: edad superior a 18 años, cardiopatía de origen no isquémico e historia de insuficiencia cardíaca. Se excluyó a los pacientes con infección, enfermedad inflamatoria crónica, tumor maligno e insuficiencia renal (creatinina $>2 \mathrm{mg} / \mathrm{dl}$ ), así como a las mujeres con posibilidad de embarazo. El estudio fue aprobado por el comité ético de la institución y todos los pacientes firmaron el consentimiento informado antes de ser incluidos.

\section{Estudios}

En todos los pacientes se realizaron una historia clínica completa, una exploración física, análisis habituales (hemograma, sistemática de orina y bioquímica), electrocardiograma de 12 derivaciones y ecocardiograma. Todos tenían una angiografía coronaria efectuada por diversos motivos en los últimos 6 meses.

\section{Tratamiento}

Cuando se efectuó el estudio los pacientes estaban recibiendo tratamiento con los siguientes grupos de fármacos: inhibidores de la enzima de conversión de la angiotensina (IECA) o de los receptores de la angiotensina II (ARA-II), digital, diuréticos y bloqueadores beta. Cada paciente recibía varios de estos fármacos y algunos pacientes los recibían todos.

\section{Tomografía por emisión de positrones}

Se hicieron 80 estudios en 40 pacientes: en cada uno de ellos, la PET se llevó a cabo con N-13 amonio en reposo (basal) e inmediatamente después de la infusión de trifosfato de adenosina como estrés farmacológico $^{1}$.

\section{Neurohormonas y citocinas}

Se practicó una punción venosa para la extracción de sangre en ayunas y después de 30 min de reposo en decúbito supino. Todos los tubos contenían anticoagulante EDTA. Las muestras fueron centrifugadas durante 30 min y el plasma almacenado a $-80{ }^{\circ} \mathrm{C}$ para su posterior análisis.

Las neurohormonas se determinaron mediante radioinmunoanálisis siguiendo las instrucciones del fabricante (IRMA, CIS bio-international, Francia). Los valores normales de nuestro laboratorio, previamente establecidos con un grupo control, son: noradrenalina, $273 \pm 102 \mathrm{pg} / \mathrm{ml}$; endotelina- $1,1,76 \pm 0,8 \mathrm{pg} / \mathrm{ml}$; angiotensina II, $6,1 \pm 3 \mathrm{pg} / \mathrm{ml}$; ANP, $19 \pm 5 \mathrm{pg} / \mathrm{ml} \mathrm{y}$ BNP, $17,4 \pm 4,8 \mathrm{pg} / \mathrm{ml}$. Las citocinas se determinaron mediante enzimoinmunoanálisis siguiendo las instrucciones del fabricante (RD System, Abingdon, Reino Unidos). Los valores normales son: factor de necrosis tumoral alfa, $3,1 \pm 0,6 \mathrm{pg} / \mathrm{ml} ; \mathrm{IL}-1 \beta, 0,5 \pm 0,4 \mathrm{pg} / \mathrm{ml}$; receptor soluble de la IL-2, $1.007 \pm 250 \mathrm{pg} / \mathrm{ml}$ e IL-6, $1,77 \pm 0,9 \mathrm{pg} / \mathrm{ml}$.

\section{Análisis estadístico}

Los datos del estudio se recogieron en una base de datos SPSS. La estadística descriptiva se expresa como media \pm desviación estandar (DE). La correlación entre las variables cuantitativas se examinó mediante el coeficiente de Pearson si la distribución era normal o el de Spearman si no lo era. Se consideró que había significación estadística con un valor de $\mathrm{p}<0,05$.

\section{RESULTADOS}

\section{Características de los pacientes estudiados}

En la tabla 1 se muestran las características demográficas y los factores de riesgo cardiovascular. 
TABLA 1. Características de los pacientes

\begin{tabular}{lc}
\hline Número de pacientes & 40 \\
Edad, años, media \pm DE & $63 \pm 11$ \\
Varones, $n(\%)$ & $27(68)$ \\
IMC, media \pm DE & $27,7 \pm 4,4$ \\
Tabaquismo, n (\%) & $5(12,5)$ \\
Hipertensión arterial, n (\%) & $24(60)$ \\
Diabetes mellitus, $\mathrm{n}(\%)$ & $6(15)$ \\
CT/cHDL, media $\pm \mathrm{DE}$ & $5,75 \pm 1,37$ \\
\hline
\end{tabular}

CT/CHDL: colesterol total/colesterol unido a lipoproteínas de alta densidad; DE: desviación estándar; IMC: índice de masa corporal.

TABLA 2. Diagnóstico y variables ecocardiográficas

\begin{tabular}{lc}
\hline & Número \\
\hline Miocardiopatía dilatada, $\mathrm{n}(\%)$ & $24(60)$ \\
Valvulopatía mitral-aórtica, $\mathrm{n}(\%)$ & $15(37,5)$ \\
Miocardiopatía restrictiva, $\mathrm{n}(\%)$ & $1(2,5)$ \\
Fracción de eyección, \%, media $\pm \mathrm{DE}$ & $41 \pm 15$ \\
Diámetro telediastólico VI, mm & $62 \pm 13$ \\
Indice de VTD VI, ml/m², media $\pm \mathrm{DE}$ & $113 \pm 53$ \\
\hline
\end{tabular}

VI: ventrículo izquierdo; VTD: volumen telediastólico; DE: desviación estándar.

TABLA 3. Valores de neurohormonas

\begin{tabular}{lcc}
\hline Neurohormonas & Pacientes & Valores de referencia \\
\hline ET-1 & $19 \pm 26$ & $1,76 \pm 0,8$ \\
AG-II & $27 \pm 38$ & $6,1 \pm 3$ \\
ANP & $74 \pm 86$ & $19 \pm 5$ \\
BNP & $137 \pm 210$ & $17,4 \pm 4,8$ \\
\hline
\end{tabular}

AG-II: angiotensina II; ANP: péptido natriurético auricular; BNP: péptido natriurético cerebral; ET-1: endotelina-1.

Todos los valores se expresan en $\mathrm{pg} / \mathrm{ml}$.

En la tabla 2 se muestran el diagnóstico y las principales variables ecocardiográficas de los pacientes cuando se hizo el estudio.

\section{Neurohormonas y citocinas}

Los valores medios de las neurohormonas medidas se muestran en la tabla 3 y los valores medios de las citocinas medidas se muestran en la tabla 4 . Se aprecia en la tabla 3 que todas las neurohormonas medidas estuvieron elevadas respecto a los valores de referencia. En la tabla 4 se observa que todas las citocinas medidas estuvieron elevadas respecto a los valores de referencia, excepto la IL-1 $\beta$.

\section{Reserva de flujo coronario medida por PET}

Estos valores se resumen en la tabla 5. Teniendo en cuenta que la RFC normal en individuos de edad similar a los de este estudio es $3 \pm 0,73^{12}$, se aprecia que en el conjunto de pacientes está considerablemente reducida.
TABLA 4. Valores de citocinas

\begin{tabular}{lcc}
\hline & Pacientes & Valores de referencia \\
\hline TNF- $\alpha$ & $24,4 \pm 1,99$ & $3,1 \pm 0,6$ \\
IL-1 $\beta$ & $0,34 \pm 0,56$ & $0,5 \pm 0,4$ \\
RsIL-2 & $1.647 \pm 799$ & $1.007 \pm 250$ \\
IL-6 & $24,6 \pm 3,2$ & $1,77 \pm 0,9$ \\
\hline
\end{tabular}

IL-1ß: interleucina 1ß; IL-6: interleucina-6; RsIL-2: receptor soluble de la interleucina-2; TNF- $\alpha$ : factor de necrosis tumoral alfa.

Los valores se expresan en $\mathrm{pg} / \mathrm{ml}$.

TABLA 5. Flujo y reserva medidos con tomografía por emisión de positrones

\begin{tabular}{ll}
\hline $\mathrm{FM}_{\mathrm{b}}\left(\mathrm{ml} \cdot \mathrm{g}^{-1} \cdot \mathrm{min}^{-1}\right)$ & $0,76 \pm 0,26$ \\
$\mathrm{FM}_{\text {ATP }}\left(\mathrm{ml}^{-1} \cdot \mathrm{gin}^{-1}\right)$ & $1,43 \pm 0,77$ \\
$\operatorname{RFC}\left(\mathrm{FM}_{\text {ATP }} / \mathrm{FM}_{\mathrm{b}}\right)$ & $1,87 \pm 0,88$ \\
\hline
\end{tabular}

ATP: trifosfato de adenosina; b: basal; FM: flujo miocárdico; RFC: reserva de flujo coronario.

TABLA 6. Correlación entre reserva, neurohormonas y citocinas

\begin{tabular}{lcc}
\hline & Coeficiente de correlación & $\mathbf{p}$ \\
\hline ET-1 & $-0,100$ & 0,551 \\
AG-II & $-0,045$ & 0,790 \\
ANP & $-0,476$ & 0,003 \\
BNP & $-0,442$ & 0,005 \\
TNF- $\alpha$ & 0,73 & 0,678 \\
IL-1 $\beta$ & 0,009 & 0,960 \\
RsIL-2 & $-0,97$ & 0,580 \\
IL-6 & $-0,509$ & 0,002 \\
\hline
\end{tabular}

AG-II: angiotensina II; ANP: péptido natriurético auricular; BNP: péptido na-

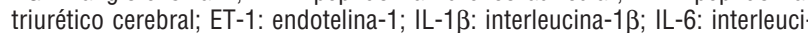
na-6; NA: noradrenalina; TNF- $\alpha$ : factor de necrosis tumoral alfa; RsIL-2: receptor soluble de la interleucina-2.

Todos los valores se expresan en $\mathrm{pg} / \mathrm{ml}$.

\section{Correlación entre reserva de flujo coronario, neurohormonas y citocinas}

Como se observa en la tabla 6, se encontró una correlación significativa negativa entre la RFC y los siguientes factores: ANP, BNP e IL-6. No se encontró correlación con los restantes factores.

\section{DISCUSIÓN}

\section{Neurohormonas}

El presente estudio demuestra una activación neurohormonal en la insuficiencia cardíaca, con elevación de todos los factores medidos respecto a los valores de referencia. La noradrenalina es el factor menos elevado, lo cual puede deberse a los fármacos que recibían estos pacientes, algunos de ellos (bloqueadores beta) con efecto inhibidor del sistema simpático. La angio- 
tensina II está elevada a pesar de que la mayoría de los pacientes estaban siendo tratados con IECA o ARA-II. Probablemente, la elevación habría sido mayor sin estos fármacos.

\section{Citocinas}

De entre las determinadas, el TNF- $\alpha$, la IL-6 y el receptor soluble de la IL-2 estuvieron elevados en los pacientes con respecto a los valores de referencia. Estos resultados concuerdan con los referidos por la mayoría de los autores, que han encontrado activación neurohormonal y elevación de citocinas en la insuficiencia cardíaca ${ }^{2-11}$.

Sin embargo, no se encontró elevación en la IL-1ß. Esta citocina desempeña un importante papel fisiopatológico en la génesis y el desarrollo de la insuficiencia cardíaca pero, a diferencia de otras citocinas, sus acciones se centran fundamentalmente en el miocardio ${ }^{13}$. Esto explicaría el fracaso de la mayoría de los estudios $^{14}$, incluido el nuestro, en demostrar valores elevados de IL-1 $1 \beta$ en sangre periférica.

La IL-6 es la citocina que más se ha elevado en nuestro estudio, lo cual concuerda con multitud de trabajos que demuestran un papel fundamental de la IL-6 en el ámbito de la insuficiencia cardíaca ${ }^{2,9,15,16}$.

\section{Reserva de flujo coronario medida con PET en la insuficiencia cardíaca}

Los pacientes con insuficiencia cardíaca no isquémica tienen disminuida la RFC. La reserva presenta la máxima disminución en los sujetos en clase funcional más avanzada (III-IV) ${ }^{1}$, con diferencias significativas respecto a los que están en clase I. En el estudio actual se presentan los valores medios del grupo total de 40 pacientes sin dividirlos en grupos, con objeto de simplificar los datos.

\section{Correlación entre reserva de flujo coronario, neurohormonas y citocinas}

El presente estudio muestra una correlación negativa significativa entre la RFC medida por PET y diversos factores (ANP, BNP e IL-6) en pacientes con insuficiencia cardíaca no isquémica. Esperábamos encontrar una correlación negativa entre la RFC y algunas neurohormonas y citocinas que producen vasoconstricción, como noradrenalina, angiotensina II, endotelina-1 y factor de necrosis tumoral alfa (TNF- $\alpha$ ), pero no fue así.

Se sabe que los péptidos natriuréticos tienen propiedades vasodilatadoras, tanto arteriales como venosas y, por tanto, ejercen un efecto compensador en la insuficiencia cardíaca ${ }^{17}$. La IL-6 aumenta la vasodilatación dependiente del endotelio a la vez que induce disfunción miocárdica, lo que sugiere que su producción local regula la resistencia y la permeabilidad vascular en estos pacientes ${ }^{18}$. Es bien conocido que en la insuficiencia cardíaca se produce disfunción endotelial ${ }^{19}$, con vasoconstricción de grandes y pequeños vasos en el miocardio ${ }^{20}$ y en las arterias periféricas ${ }^{21}$. Esto puede deberse no sólo a la activación simpática, incremento de endotelina-1 y TNF- $\alpha$, sino también a otros mecanismos, como puede ser la producción aumentada de radicales libres de oxígeno ${ }^{19,22,23}$. La elevación de ANP, BNP e IL-6 puede producirse como mecanismo de compensación que trata de contrarrestar la vasoconstricción debida a múltiples causas.

En nuestro conocimiento, ningún estudio previo ha evaluado la correlación entre RFC medida por PET, neurohormonas y citocinas en la insuficiencia cardíaca. Como ocurre en tantos procesos, algunos mecanismos de compensación resultan deletéreos, como es la elevación de la IL-6, que puede aumentar la disfunción ventricular y, por tanto, empeorar la insuficiencia cardíaca.

\section{Limitaciones}

La principal limitación se debe al escaso número de pacientes incluidos en el estudio, debido a la dificultad que conlleva la PET. Se trata de una exploración compleja y de coste elevado, por lo que no se puede realizar a muchos pacientes como habría sido deseable. Con los datos presentados no se puede saber si un mayor número de casos permitiría encontrar mayor número de relaciones significativas que las observadas en este estudio.

\section{CONCLUSIONES}

La disminución de la RFC que se observa en la insuficiencia cardíaca se correlaciona con la elevación de determinados factores neurohormonales (ANP, BPN) y citocinas (IL-6), todos ellos con efecto vasodilatador. Se trata probablemente de mecanismos de compensación que resultan insuficientes ante la disfunción endotelial que presentan estos enfermos por múltiples factores.

\section{BIBLIOGRAFÍA}

1. Coma-Canella I, García-Velloso MJ, Macías A, Villar L, CosínSales J, Martí-Climent JM, et al. Disminución de la reserva de flujo coronario en pacientes con insuficiencia cardíaca no isquémica. Rev Esp Cardiol. 2003;56:354-40.

2. Vidal B, Roig E, Pérez-Villa F, Orús J, Pérez J, Jiménez V, et al. Valor pronóstico de los niveles de citocinas y neurohormonas en la insuficiencia cardíaca severa. Rev Esp Cardiol. 2002;55:481-6.

3. Francis GS, Cohn JN, Johnson G, Rector TS, Goldman S, Simon A. Plasma noradrenaline, plasma renin activity and congestive heart failure: relations to survival and the effects of therapy in VHeFT II. Circulation. 1993;87 Suppl VI:40-8. 
4. Francis G. The relationship of the sympathetic nervous system and the renin-angiotensin system in congestive heart failure. Am Heart J. 1989;118:642-8.

5. Wei CM, Lerman A, Rodeheffer RJ, McGregor CG, Brandt RR, Wright $\mathrm{S}$, et al. Endothelin in human congestive heart failure. Circulation. 1994;89:1580-6.

6. Winters TJ, Sallman AL, Baker BJ, Meadows J, Rico DM, Vesely DL. The N-terminus and 4000 molecular weight peptide from the mid portion of the $\mathrm{N}$-terminus of the atrial natriuretic factor prohormone each circulate in humans and increase in congestive heart failure. Circulation. 1989;80:438-49.

7. Davis M, Espiner E, Richards G, Bilings J, Town I, Neil A. Plasma brain natriuretic peptide in assesment of acute dyspnoea. Lancet. 1994;343:440-4.

8. Testa M, Yeh M, Lee P, Fanelli R, Loperfido F, Berman JW, et al. Circulating levels of cytokines and their endogenous modulators in patient with mild to severe congestive heart failure due to coronary artery disease or hypertension. J Am Coll Cardiol. 1996;28:964-71.

9. Roig E, Orus J, Pare C, Azqueta M, Filella X, Pérez-Villa F, et al. Serum interleukin- 6 in congestive heart failure secondary to idiopathic dilated cardiomyopathy. Am J Cardiol. 1998;82:688-90.

10. Limas CJ, Goldenberg IF, Limas C. Soluble interleukin-2 receptor in patients with dilated cardiomyopathy. Correlation with disease severity and cardiac autoantibodies. Circulation. 1995;91:631-4.

11. Bristow MR. Tumor necrosis factor-alpha and cardiomyopathy. Circulation. 1998;97:1340-1.

12. Czernin J, Muller P, Chan S, Brunken RC, Porenta G, Krivokapich $\mathrm{J}$, et al. Influence of age and hemodynamics on myocardial blood flow and flow reserve. Circulation. 1993;88:62-9.

13. Baumgarten G, Knuefermann P, Mann DL. Cytokines as emerging targets in the treatment of heart failure. Trends Cardiovasc Med. 2000;10:216-23.
14. Aukrust P, Ueland T, Lien E, Bendtzen K, Muller F, Andreassen $\mathrm{AK}$, et al. Cytokine network in congestive heart failure secondary to ischemic or idiopathic dilated cardiomyopathy. Am J Cardiol. 1999;83:376-82.

15. Suzuki H, Sato R, Sato T, Shoji M, Iso Y, Kondo T, et al. Timecourse of changes in the levels of interleukin 6 in acutely decompensated heart failure. Int J Cardiol. 2005;100:415-20.

16. Hirota H, Izumi M, Hamaguchi T, Sugiyama S, Murakami E, Kunisada $\mathrm{K}$, et al. Circulating interleukin- 6 family cytokines and their receptors in patients with congestive heart failure. Heart Vessels. 2004;19:237-41.

17. Abassi Z, Karram T, Ellaham S, Winaver J, Hoffman A. Implications of the natriuretic peptide system in the pathogenesis of heart failure: diagnostic and therapeutic importance. Pharmacol Ther. 2004;102:223-41.

18. Tsutamoto T, Hisanaga T, Wada A, Maeda K, Ohnishi M, Fukai $\mathrm{D}$, et al. Interleukin-6 spillover in the peripheral circulation increases with the severity of heart failure, and the high plasma level of interleukin-6 is an important prognostic predictor in patients with congestive heart failure. J Am Coll Cardiol. 1998;31:391-8.

19. Fang ZY, Marwick TH. Vascular dysfunction and heart failure: epiphenomenon or etiologic agent? Am Heart J. 2002;143:383-90.

20. Mathier MA, Rose GA, Fifer MA, Miyamoto MI, Dinsmore RE, Castano HH, et al. Coronary endothelial dysfunction in patients with acute-onset idiopathic dilated cardiomyopathy. J Am Coll Cardiol. 1998;32:216-24.

21. Katz SD. The role of endothelium-derived vasoactive substances in the pathophysiology of exercise intolerance in patients with congestive heart failure. Prog Cardiovasc Dis. 1995;26 Suppl 2:9-12.

22. Sharma R, Davidoff MN. Oxidative stress and endothelial dysfunction in heart failure. Congest Heart Fail. 2002;8:165-72.

23. Bauersachs J, Schafer A. Endothelial dysfunction in heart failure: mechanisms and therapeutic approaches. Curr Vasc Pharmacol. 2004;2:115-24. 